Recibido: 19-07-2019/ Revisado: 26-07-2019/Aceptado: 19-08-2019/ Publicado: 06-09-2019

DOI: https://doi.org/10.33262/exploradordigital.v3i3.1.893

\title{
La neuroeducación y su contribución al aprendizaje de
} las matemáticas

\section{Neuroeducation and its contribution to the learning of mathematics}

Javier Roberto Mendoza Castillo. ${ }^{1}$, Rómel Manolo Insuasti. ${ }^{2}$ Jenner José Baquero Luna. ${ }^{3}$

\begin{abstract}
Today the study of neuroscience and its contribution to the study of various disciplines has increased, especially in the field of education as a process that allows explaining and understanding how the brain acts before certain stimuli. Neuroeducation based on the analysis of the mind, the brain and its articulation to the field of primary education resulting in order to adapt, teaching methods and learning techniques that facilitate this process. In this sense, the present study allows us to respond because in certain individuals mathematical learning is easier than in others
\end{abstract}

Keywords: Neuroeducation, mathematics, brain, neurosciences, learning

\section{Resumen}

Hoy en día el estudio de la neurociencia y su contribución en el estudio de varias disciplinas se ha incrementado, especialmente en el campo de la educación como un proceso que permite explicar y comprender como actúa el cerebro antes ciertos estímulos. La neuroeducación basada en el análisis de la mente, el cerebro y su articulación al campo de la educación resulta primordial a fin de adecuar, métodos y técnicas de enseñanza aprendizaje que facilite este proceso. En este sentido el presente estudio permite dar respuesta porque en ciertos individuos resulta más fácil el aprendizaje matemático que en otros.

Palabras claves: neuroeducación, matemáticas, cerebro, neurociencias, aprendizaje

\footnotetext{
1 Escuela Superior Politécnica de Chimborazo, Facultad de Ciencias Pecuarias, Chimborazo, Ecuador jmendoza@espoch.edu.ec

${ }^{2}$ Escuela Superior Politécnica de Chimborazo, Facultad de Mecánica, Chimborazo, Ecuador. rinsuasti@espoch.edu.ec

3 Escuela Superior Politécnica de Chimborazo, CIDED, Facultad de Ciencias, Chimborazo, Ecuador, jose.baquero@espoch.edu.ec
} 


\section{Introducción}

\section{La neurociencia y su aporte al proceso de enseñanza aprendizaje}

La neurociencia estudia el funcionamiento cerebral para comprender los procesos mentales, particularmente los más complejos como la inteligencia, la conciencia, la personalidad o las emociones. (Ustárroz 2011).

Por su parte Carles (2004), define a la neurociencia como la disciplina que busca entender cómo la función cerebral da lugar a las actividades mentales, tales como la percepción, la memoria, el lenguaje e incluso la conciencia. La tarea central de la neurociencia es la de intentar explicar cómo es que actúan millones de células nerviosas individuales en el encéfalo para producir una conducta y cómo, a su vez, estas células están influenciadas por el medioambiente, (Jessel, et al. 1997), citado por (Barrera, 2009). Las neurociencias pueden realizar importantes contribuciones al conocimiento para facilitar la comprensión de los procesos cognitivos claves para la enseñanza-aprendizaje, tales como la memoria, la atención, el lenguaje, la lectoescritura, las funciones ejecutivas, la toma de decisiones, la creatividad y la emoción, entre otros. (Manes 2018)

Autores como (Tomlinson \& Kalbfleisch, 1998, Goswami 2004, Mcnab 2009, Campos 2011) coinciden en que la comprensión de la relación cerebro-aprendizaje permitirá al educador entender cómo funciona el cerebro, cómo procesa la información, cómo controla las emociones, cómo este aprende, registra, conserva y evoca una información, de esta información se contará con el insumo necesario para poder desarrollar las mejores estrategias que contribuyan a un mejor aprendizaje y a una autorregulación de las mismas.

Para (Rosler 2018) una enseñanza menos superficial debería tener como eje las emociones, la pasión, el deseo, la motivación intrínseca, el humor, el aprendizaje en contexto -fuera del aula- y el aprender con el cuerpo, esta sería la piedra angular para que a partir de este conocimiento se pueda mejorar los métodos y experiencias de aprendizaje, convirtiendo el salón de clases en una vivencia de experiencias más motivadoras y emocionantes.

De lo anterior se desprende que el aprendizaje es un proceso importante en el quehacer educativo que apoyado en la neurociencia permite comprender dicho proceso desde el análisis de las estructuras complejas del cerebro, lo que permite al educador alinear las 
actividades didácticas y pedagógicas de tal forma que estas despierten en el estudiante, el interés y la motivación por aprender. A esa relación entre aprendizaje, enseñanza y neurociencia diversos autores la han llamado neuroeducación, entendida como el desarrollo de la neuro mente durante la escolarización (De la Barrera, 2009).

\section{Cerebro y pensamiento matemático}

La matemática es una actividad mental, independiente de la experiencia. El matemático trabaja a partir de definiciones y axiomas y llega a verdades. No obstante, podemos interactuar con el mundo físico mediante el conocimiento que acumulamos por la actividad matemática. (Bravo, 2016).

La actividad matemática según algunas teorías se tiene en el lóbulo frontal y parietal del cerebro, las matemáticas son una actividad mental que lleva una verdad, la misma que debido a la evolución se adapta a varias realidades como una modernización o resurgimiento, aquí se entiende que debido a este cambio propio de las matemáticas se han identificado nuevos campos de investigación matemática dando paso a la matematización. (Fernández, 2010)

La mayoría de personas creen que las matemáticas son complejas, que para ellos no son buenas, sin embargo, estudios han revelado que tanto el pensamiento como los resultados que ellos implican se pueden cambiar. (Ashcraft, 2002)

Dweck (2008) manifiesta que las matemáticas no se consideran un don, basta creer en sí mismo teniendo una mente abierta y progresiva se logrará un resultado matemático eficiente. Las mismas conexiones neuronales que los adultos utilizan para resolver cálculos complejos están presentes y activas desde por lo menos los cuatro años, lo que indica que desde pequeños nos estamos preparando para manejar información cuantitativa toda la vida. (Gramling, 2006)

Los seres humanos nacen con un sentido numérico inherente, desde niños se logra distinguir operaciones con diferentes objetos esto demuestra que en un campo un poco rudimentario desde pequeños experimentamos un conocimiento básico de los números naturales, lo que demuestra que la capacidad de aprendizaje no se ve limitada en tempranas edades.

Nuestro cerebro en si prefiere lo más concreto, lo que le ayude a resolver sus problemas de una manera más fácil, el cerebro cambia se modifica según la sinapsis1 que conectan a las 
neuronas que están en actividad desarrollando una regeneración neuronal, la que permite una buena contribución al cerebro evitando enfermedades neurodegenerativas, y también en un tratamiento para algunas de ellas. Si algo ha demostrado la ciencia es que mientras más ejercitemos nuestro cerebro especialmente con cálculos y operaciones matemáticas, mejora la capacidad de concentración, aumenta la flexibilidad cognitiva, y mejora la memoria a largo plazo.

En el cerebro la actividad mental dentro de las matemáticas trabaja a partir de las siguientes definiciones:

Adaptación: es el conocimiento matemático que se posee se aplica a la realidad objeto de estudio o contribuye a su desarrollo.

Modelización La matemática estudia la realidad, creando modelos a partir del conocimiento matemático que se posee.

Resurgimiento El conocimiento matemático se reconoce en el comportamiento de realidades.

Hay que tener en cuenta que en varias ocasiones el proceso de la resolución matemática puede realizarse por más de un método. Esto es debido, tanto a la propia evolución de la matemática como a la evolución de la ciencia, interviene en la interacción con la realidad objeto de estudio.

\section{Una nueva ciencia para el aprendizaje matemático}

Hoy en día es necesario mencionar que, desde la mitad del siglo XX hasta nuestros días los avances mostrados por las ciencias de la educación que comprende el aprendizaje del ser humano son indudables. Sin embargo, aún no se puede decir, con certeza, que estos avances se inserten con fundamento y rigor científico. Aún se está lejos de hacer predicciones y controlar los aprendizajes que se manifiestan en los estudiantes, así como de dotar de capacidad suficiente a docentes basados en resultados aislados del que enseña. He aquí un desafío a enfrentar las ciencias de la educación para este siglo (Waldegg, 2003, pág. 82)

No obstante, existen estudios como el de Qin (20014), que demuestran que la receptividad más grande del cerebro es en la adolescencia siendo propicio para la enseñanza del álgebra así también Luna (2004) coincide con lo planteado con este autor, al manifestar que, como 
adultos, estaríamos limitados en nuestra habilidad de "aprender" no así en la adolescencia, resultados que se obtuvieron mediante el uso con equipos biométricos.

La neurociencia está aportando respuestas a situaciones de sumo interés para los docentes, por ejemplo; existen investigaciones donde se evidencia que tanto un cerebro en maduración como uno ya maduro se modifican estructuralmente cuando ocurre un evento de aprendizaje. (Bransford, 2003)

Si bien es cierto ha proliferado el interés por este tipo de estudios, para (Waldegg, 2003) existe una nueva frontera, donde se vislumbran formas innovadoras de soportar las hipótesis sobre el aprendizaje, basadas en datos reales y puros, provenientes de los resultados obtenidos de acuerdo con el funcionamiento del cerebro. Según el razonamiento de esta investigadora, la psicología cognoscitiva debe interactuar e interrelacionarse con otras disciplinas para seguir aportando soluciones al aprendizaje.

La expectativa para realizar nuevos estudios del cerebro, la emocionalidad vuelve a ocupar estudios importantes especialmente, en la enseñanza de las matemáticas, colocando la racionalidad platónica cartesiana en una disyunción epistémica. (Coleman, 2000) este autor afirma que el aprendizaje no está separado de las emociones. Instruir a las personas en descubrir sus emociones es tan significativo para la enseñanza y el aprendizaje, sin emoción no existe atención y por lo tanto esto dificulta el aprendizaje.

Para muchos investigadores la definición de matemáticas hace referencia a la memoria de trabajo duro (Baddeley, 1997) menciona que es el sistema con un nivel funcional limitado, pero con capacidad para almacenar, manipular las informaciones, permitiendo así la ejecución de tareas cognitivas tales como el razonamiento, la comprensión y la resolución de problemas, soportadas por el mantenimiento y disponibilidad de esas informaciones. Para el aprendizaje la atención siempre es un papel fundamental. Pero la atención es proceso sensible y necesita conectar con emociones entre el receptor y emisor

Así como el descubrimiento de nuevas experiencias para que la atención sea mantenida dentro de la educación matemática la cual es una ciencia exacta, resulta primordial captar la atención de todos aquellos que comparten los conocimientos del saber, plantear estrategias para motivar a estudiantes y así conducirlos a un mejor aprendizaje. Gallese y Lakoff, (2005), 
plantean que un saber debe estar unido de manera íntima al funcionamiento de nuestro sistema sensorio-motor. Este nuevo enfoque, da pie a una nueva teoría de aprendizaje en la que pensamos no sólo con la ayuda del lenguaje y de los símbolos, sino también a través de los sentidos. Aprender a enseñar desde el cerebro del que aprende.

Los docentes de hoy en un futuro, sin lugar a dudas, soportarán su didáctica en las investigaciones relacionadas con las neurociencias. Un paradigma que surge con intensidad y marcará un hito tanto en la enseñanza como en el aprendizaje.

\section{Metodología}

Revisión analítica descriptiva de fuentes bibliográficas y documentales, identificación de las aproximaciones teóricas elaboradas sobre el tema. Conocer las aproximaciones metodológicas del estudio del tema, identificar las variables asociadas al estudio del tema

\section{Conclusiones}

- Como hemos visto en este estudio la matemática es una actividad mental que trabaja con definiciones y axiomas lo que no permite interactuar con el medio físico de una manera apropiada, por lo tanto, se hace necesario interactuar el aprendizaje matemático con la psicología cognoscitiva para encontrar soluciones al aprendizaje, teniendo presente a la neurociencia.

- La neurociencia, al estudiar el funcionamiento cerebral necesario para comprender los procesos mentales como la inteligencia, la conciencia, la personalidad o las emociones, es fundamental para el aprendizaje que permite mejorar los métodos y técnicas neurológicas utilizadas para este fin.

- El aprendizaje al ser un proceso importante en el proceso educativo debe estar apoyado en la neurociencia, para facilitar la comprensión de las matemáticas, de esta manera el educador planteará actividades didácticas y pedagógicas de tal forma que despierte el interés del estudiante y su motivación por aprender. 


\section{Referencias bibliográficas}

Ashcraft, M. (2002). Math anxiety: personal, educational, and Cognitive consequences. Current Directions in Psuchological Science., 5.

Baddeley, A. (1997). Human Memory. Theory and Practice. New York: Mc Graw Hill. Battro, A., \& Cardinali, D. (1996). Mas cerebro en la educacion. Argentina: La Nacion. Bransford, J. B. (2003). How People Learn: Brain, Mind, Experience, and School. Estados Unidos: National Academy Press. National Academy Press.: Press.

Carles, E. (2004). Aproximación histórica y conceptual a la Neurociencia Cognitiva. Cognitiva, 141-162.

Coleman, D. (. (2000). La Inteligencia Emocional. ¿Por qué es más importante que el cociente intelectual? España: Vergara.

Educarchile. (2011). Metodo singapur en enseñanza matematica. Chile : Educarchile.

Espinosa, T., \& Tracely, N. (2008). the scientifically substantiated art of teaching: a study in the development of standards in the new academic field of neuroeducation (Mind, Brain, and Education Science). New York: Capella University.

Fernández, J. (2010). Neurociencias y Enseñanza de la Matemática. Iberoamericana de Educacion, 2.

Geake, J. (2002). The Gifted Brain. Obtenido de http://www.edfac.unimelb.edu.au/LED/GCE/brain.html

Gallese, V. \& Lakoff, G. (2005). The brain's concepts: the role of the sensory-motor system in conceptual knowledge. Cognitive Neuropsychology 22 (3/4), 455-479

Hart, L. (1998). Human Brain \& human learning. New York: Basic Books. doi:ISBN: 058288-379-5

Jimenez, C. A. (2006). El efecto Mozart.

Kalbfleisch, M. (2012). Neurotechnology in education: Intervening, enabling, ¿or enhancing? In J. Giordano (Ed.), Neurotechnology: Premises, potential and problems (A volume in the advances of neurotechno-logy: Ethical, legal, and social issues series). Taylor \& Francis Eds.New York (USA).

Kandel, E., Schwartz, J., \& Jessell, T. (1997). Neurociencia y conducta. Madrid: Prentice Hall.

La revolución de las matemáticas. (2011). el método singapur. Chile: Revista Educar.

McLean, P. (1998). Evolución del Cerebro Triuno. New York: editorial pleumpress.

Qin, Y., Carter, C. S., Silk, E. M., Stenger, V. A., Fissell, K., Goode, A. \& Anderson, J.R. (2004). The change of the brain activation patterns as children learn algebra equation solving. Proceedings of the National Academy of Sciences of the United States of America, 101(15), 5686-5691.

Sousa, D. (2014). Neurociencia Educativa. Madrid, España: Narcea, S.A. doi: ISBN: 97884- 277- 2029- 9

Tirapu, J. (2011). Neuropsicología - neurociencia y las ciencias "PSI" Cuadernos de Neuropsicología / Panamerican Journal of Neuropsychology, vol. 5, núm. 1, julio, 
2011, pp. 11-24 Centro de Estudios Académicos en Neuropsicología Rancagua, Chile

Willis, S. (2007). Creating a knowledge base for teaching: A conversation with James Stigler. San Francisco: Educational Leadership. 
Para citar el artículo indexado.

Mendoza Castillo, J., Insuasti, R., \& Baquero Luna, J. (2019). La neuroeducación y su contribución al aprendizaje de las matemáticas. Explorador Digital, 3(3.1), 246-254. https://doi.org/10.33262/exploradordigital.v3i3.1.893

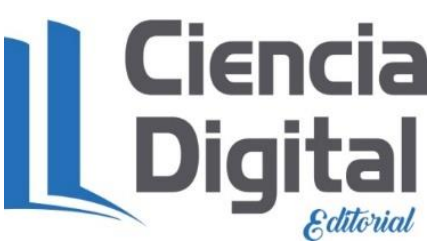

El artículo que se publica es de exclusiva responsabilidad de los autores y no necesariamente reflejan el pensamiento de la Revista Explorador Digital.

El articulo queda en propiedad de la revista y, por tanto, su publicación parcial y/o total en otro medio tiene que ser autorizado por el director o editor de la Revista Explorador

Digital.
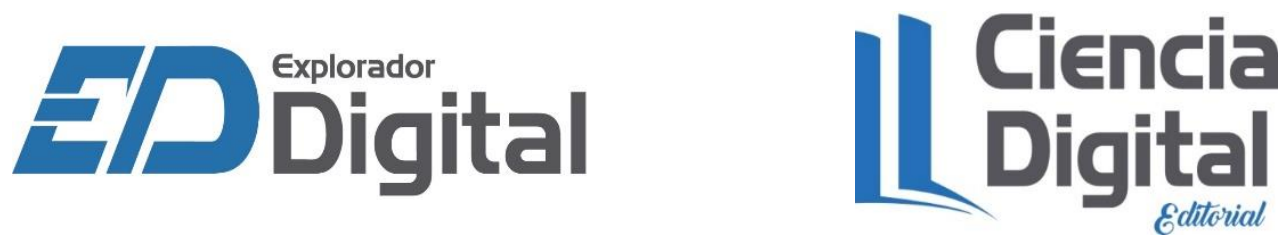